\title{
Requirement of the Composition of RNA-RNA Duplex for the Ribozyme Activity
}

\author{
Bongrae Cho \\ Department of Applied Chemistry, Cheongju University, Cheongju 360-764, Korea. E-mail: brcho@cju.ac.kr \\ Received December 23, 2009, Accepted January 15, 2010
}

Key Words: RNA-RNA duplex, DNA-RNA hybrid, Ribozyme, Thiophosphorylation

The Kin.46 kinase ribozyme was selected for the transfer of the thiophosphate from ATP- $\gamma$-S to its own 5 ' hydroxyl end in the presence of oligonucleotide effector which is complementary to its 3 ' primer binding sequence (PBS) used in the amplification steps during the original selection for activity. ${ }^{1,2}$ Omitting the oligo effector reduces the observed catalytic rate constant $\left(\mathrm{k}_{\mathrm{obs}}\right)$ by $10^{3}$ to $10^{6}$-fold, indicating that the deoxyoligonucleotide effector is necessary for its full catalytic activity. The activator helix formed by the PBS and the oligo effector is connected by a 5nt "linker" region to the substrate-binding internal guide sequence and stabilizes a long-range base-paring interaction between the 5 nuclotides of the linker and those closer to the catalytic core. According to our results, the activator helix is thought to stabilize the active conformation of the ribozyme by stabilizing the interaction between the linker and complementary nucleotides within the active site. ${ }^{3,4}$

The secondary structure of RNA-DNA hybrid duplex was observed to be different from that of RNA-RNA duplex in the analyses of X-ray fiber diffraction and molecular dynamics simulation. ${ }^{5,6}$ This work was performed to monitor how much the catalytic activity of kinase ribozyme could be affected if only one RNA strand in the RNA-RNA duplex structure composed of two RNA strands was substituted for DNA, leaving a single-stranded RNA portion which plays an important role in catalytic activity in the substituted RNA strand as an RNA.

As the trans-acting Kin.46 enzyme was formed by omitting the large single-strand region which has 20 nucleotides (not shown in Fig. 1), joining the 7-mer strand to the rest of the ribozyme, two ribozyme versions were derived from the parental ribozyme by the internal truncations. One version (a ribozyme119 derivative) has four different strands; a 7 nucleotide RNA substrate (7-mer), a 30 nucleotides "upper" strand (up), a 62 nucleotides "lower" strand (lw) and an activating oligomer (AO) with 18 nucleotides, and the other version (a ribozyme103 derivative) also has four different strands but the length of a up strand and an lw strand is shorter than that of corresponding strands of the ribozyme119 derivative; a 7 nucleotide RNA substrate (7-mer), a 24 nucleotides "upper" strand (up), a 52 nucleotides "lower" strand (lw) and an activating oligomer (AO) with 18 nucleotides (Fig. 1). It was reported that the four-stranded ribozymes 119 and 103 folded predominantly into a single, active conformation after renaturation by native gel electrophoresis. ${ }^{3}$ For the comparison of the catalytic activities of the ribozyme derivatives, APM ([( $N$-acryloylamino $)$ phenyl $]$ mercuric chloride) - PAGE (polyacrylamide gel electrophoresis) was used as a useful means which analyze thiolated or thio- phosphorylated RNA through the strong interaction between mercury and sulfur (Fig. 2) ${ }^{7-9}$ The mobility of RNA that carry thiophosphate monoester is diminished, compared with nonthiophosphated one. This is the evidence of the strong interaction between mercury and sulfur. Disulfides don't interact with the mercury in the gel matrix with APM. ${ }^{8}$ Therefore, ribozyme derivatives, truncated versions of Kin. 46 were incubated

(A)

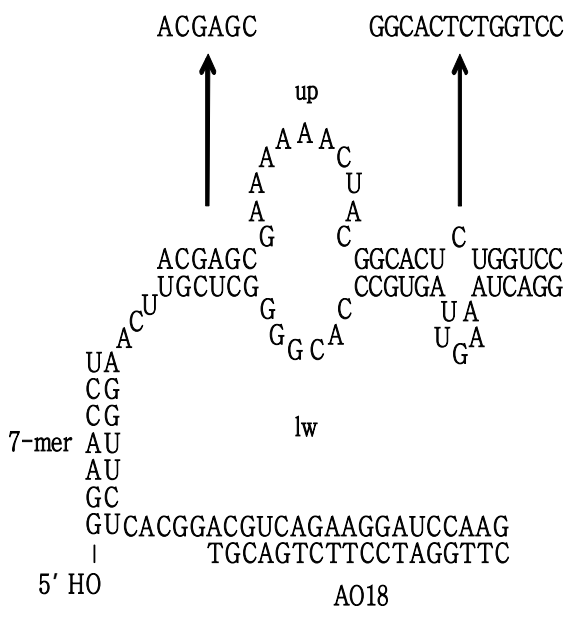

(B)

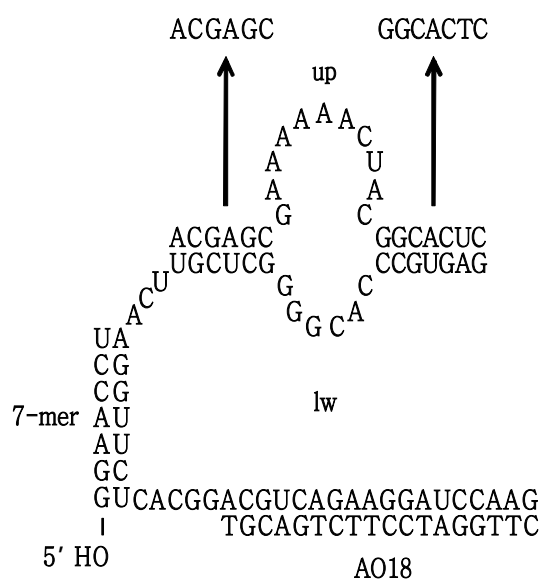

Figure 1. Secondary structures of two ribozyme versions derived from the Kin.46 self-thiophosphorylating ribozyme by the internal truncations. (A) A ribozyme119 derivative has 4 strands; a 7-mer, a upper (up) strand, a lower (lw) strand and an activating oligomer (AO) with 18 nucleotide. (B) A ribozyme103 derivative also has four different strands but the length of a up strand and an lw strand is short. Only RNA portions at the RNA-RNA double strand region in a up strand were substituted for DNA portions. 
(A)

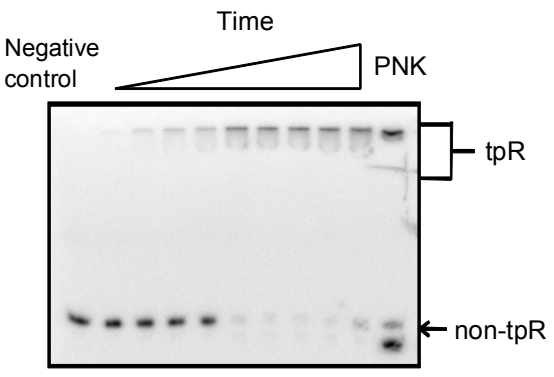

(B)

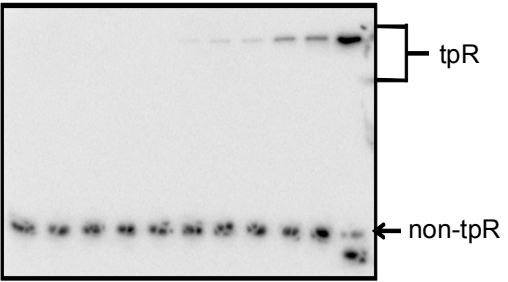

(C)

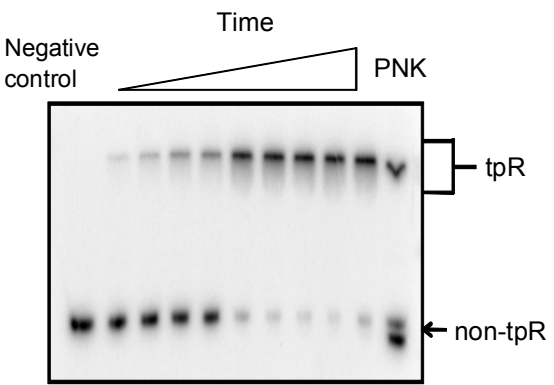

(D)

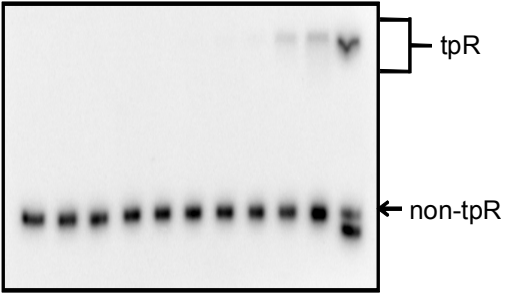

Figure 2. Kinetic assay of four-stranded ribozymes. The thiophosphorylation reaction of a ribozyme 119 composed of a 7-mer, an upper (up) strand, a lower (lw) strand and an activating oligomer (AO) 18 (A), a 7-mer, a up strand with DNA portions, an lw strand and an activating oligomer (AO) 18 (B) \{a ribozyme 103 composed of a 7-mer, a up strand, an lw strand and an activating oligomer (AO) 18 (C), and a 7-mer, a up strand with DNA portions, an lw strand and an activating oligomer (AO) 18 (D) $\}$ was initiated by addition of ATP $\gamma \mathrm{S}$ to $10 \mathrm{mM}$ at RT. Aliquots were removed at different times and the ribozymes thiophosphorylated (tpR) with ATP $\gamma \mathrm{S}$ were separated from the nonthiophosphorylated ribozymes (non-tpR) within [(N-acryloylamino) phenyl] mercuric chloride (APM) polyacrylamide gel. PNK lanes treated with polynucleotide kinase were also applied to normalize each lane.

with ATP $\gamma$ S and the products were separated by PAGE using gels that contained APM. The initial velocities for the thiophosphorylation of ribozymes with and without DNA portions in the up strand were plotted as a function of assay time. The observed rate constants are shown in Table 1. Without DNA portion, a ribozyme 119 derivative is active $\left(k_{o b s}=0.0185 \mathrm{~min}^{-1}\right)$
Table 1. Comparison of ribozyme activities

\begin{tabular}{cllll}
\hline & 7-mer & 7-mer & 7-mer & 7-mer \\
Ribozyme & up119 & up119drd & up103 & up103drd \\
Composition & lw119 & lw119 & lw103 & lw103 \\
& AO18 & AO18 & AO18 & AO18 \\
\hline $\mathrm{k}_{\text {obs }}\left(\mathrm{min}^{-1}\right)$ & 0.0185 & 0.0001 & 0.0462 & $5 \times 10^{-5}$ \\
\hline
\end{tabular}

Drd means that a up strand has DNA, RNA and DNA composition.

but the ribozyme derivative with both DNA portions at the only double strand regions and RNA portion at the only single strand region within a up strand, was barely active with a catalytic rate reduced by nearly 200 times of magnitude $\left(\mathrm{k}_{\mathrm{obs}}=\right.$ $0.0001 \mathrm{~min}^{-1}$ ) relative to the corresponding ribozyme 119 without DNA portions. This suggests that the composition of the double-stranded regions formed by the up strand and the lw strand should be not RNA-DNA hybrid but RNA-RNA duplex composition for the full catalytic activity. This decrease of the ribozyme activity was also observed in the ribozyme 103 derivative. Without DNA portion, a ribozyme103 derivative is active $\left(\mathrm{k}_{\mathrm{obs}}=0.0462 \mathrm{~min}^{-1}\right)$ but the ribozyme derivative with both DNA portions at the only double strand regions and RNA portion at the only single strand region in a up strand was barely active with a catalytic rate reduced by nearly 1,000 times of magnitude $\left(\mathrm{k}_{\mathrm{obs}}=0.00005 \mathrm{~min}^{-1}\right)$ relative to the corresponding ribozyme103 without DNA portions. X-ray fiber diffraction analyses showed that in DNA-RNA hybrid structure, the DNA chain has C-2'-endo-puckered furanose rings while the antiparallel RNA chain has C-3'-endo-puckered furanose rings. ${ }^{5,6}$ So this kinase ribozyme activity is thought to decrease because structural distortion might happen in the RNA-DNA hybrid duplex regions. But the biophysical study such as NMR (nuclear magnetic resonance) or X-ray diffraction analysis is needed for the exact explanation of the decrease of the ribozyme activity.

In summary, two ribozyme versions derived from Kin.46 self-thiophosphorylating ribozyme by internal deletions and assembled from four different strands with DNA portion at only double strand regions in an up strand, were prepared and their activities for autothiophosphorylation were compared with APM-PAGE. Two ribozyme versions with the DNA portion at double strand regions in an up strand were much less active than the corresponding ribozymes assembled with four RNA different strands.

\section{Experimental Section}

Kinetic assay of ribozyme. An internally radiolabelled lw strand using $\left[\alpha-{ }^{32} \mathrm{P}\right]$ UTP, a 7 -mer and a up strand (or a up strand with DNA portions at only double strand regions) of ribozyme, and an activating DNA oligomer were heated in $\mathrm{KCl} / \mathrm{Pipes}$ buffer $(200 \mathrm{mM} \mathrm{KCl}$ in $150 \mathrm{mM}$ Pipes- $\mathrm{KOH}, \mathrm{pH} 7.0)$ at $90^{\circ} \mathrm{C}$ for $2 \mathrm{~min}$ and allowed to cool to RT $\left(\sim 21{ }^{\circ} \mathrm{C}\right)$. These were adjusted to a final concentration of $50 \mathrm{mM} \mathrm{MgCl}$ and preincubated for $15 \mathrm{~min}$ at $\mathrm{RT}$. The thiophosphorylation reaction was initiated by addition of ATP $\gamma \mathrm{S}$ to $10 \mathrm{mM}$ at RT. Aliquots were removed at different times ( $2 \mathrm{~min}, 5 \mathrm{~min}, 8 \mathrm{~min}, 10 \mathrm{~min}$, $110 \mathrm{~min}, 230 \mathrm{~min}, 340 \mathrm{~min}, 1300 \mathrm{~min}$ and $1450 \mathrm{~min}$ ) and the 
reaction was quenched with $94 \%$ formamide, $30 \mathrm{mM}$ EDTA ( $\mathrm{pH}$ 8.0) containing xylene cyanol and bromophenol blue. Thiophosphorylated ribozymes were separated from the nonthiophosphorylated by electrophoresis in APM 6\% polyacylamide gel in $90 \mathrm{mM}$ tris-borate (pH 8.3) and $2.5 \mathrm{mM}$ EDTA containing $7 \mathrm{M}$ urea. Dried gels were exposed to storage phosphor screens and imaging (Molecular Dynamics). The extent of thiophosphorylation was calculated by dividing the radioactivity in the product band (retained at the top of the APM layer) by the sum of reacted and unreacted bands. The data were fit to a kinetic equation; The first-order rate of thiophosphorylation $\left(\mathrm{k}_{\mathrm{obs}}\right.$ : observed rate constant) was calculated by fitting to $f_{t}=\left(f_{\infty}-f_{0}\right)$ $\left(1-\exp \left(-\mathrm{k}_{\mathrm{obs}} \mathrm{t}\right)\right)$, where $\mathrm{f}_{\mathrm{t}}$ is the fraction normalized at time $\mathrm{t}$.

Acknowledgments. I thank Prof. Donald H. Burke (Univer- sity of Missouri, School of Medicine, Columbia, MO 652117310) for his helpful support.

\section{References}

1. Lorsch, J. R.; Szostak, J. W. Nature 1994, 371, 31.

2. Lorsch, J. R.; Szostak, J. W. Biochemistry 1995, 34, 15315.

3. Cho, B.; Burke, D. H. Bull. Korean Chem. Soc. 2007, 28, 463.

4. Cho, B. Bull. Korean Chem. Soc. 2007, 28, 689.

5. Arnott, S.; Chandrasekaran, R.; Millane, R. P.; Park, H. S. J. Mol. Biol. 1986, 188, 631.

6. Cheatham, T. E., III.; Kollman, P. A. J. Am. Chem. Soc. 1997, 119, 4805.

7. Igloi, G. L. Biochemistry 1988, 27, 3842.

8. Rhee, S. S.; Burke, D. H. Analytical Biochem. 2004, 325, 137.

9. Cho, B.; Burke, D. H. RNA 2006, 12, 2118. 